\title{
Analyse de l'activité de transmission écrite au cours des relèves de poste à l'hôpital
}

Évaluation ergonomique de l'usage d'un nouveau format d'écriture

Jean-Michel Boucheix et Michèle Coiron

\section{OpenEdition}

\section{Journals}

Édition électronique

URL : http://journals.openedition.org/activites/1949

DOI : 10.4000/activites. 1949

ISSN : 1765-2723

Éditeur

ARPACT - Association Recherches et Pratiques sur les ACTivités

\section{Référence électronique}

Jean-Michel Boucheix et Michèle Coiron, « Analyse de l'activité de transmission écrite au cours des relèves de poste à l'hôpital », Activités [En ligne], 5-1 | avril 2008, mis en ligne le 15 avril 2008, consulté le 10 décembre 2020. URL : http://journals.openedition.org/activites/1949 ; DOI : https://doi.org/ 10.4000/activites. 1949

\section{c) () $\Theta$}

Activités est mis à disposition selon les termes de la licence Creative Commons Attribution - Pas d'Utilisation Commerciale - Pas de Modification 4.0 International. 


\title{
Analyse de l'activité de transmission écrite au cours des relèves de poste à l'hôpital: Évaluation ergonomique de l'usage d'un nouveau format d'écriture
}

\author{
Jean-Michel Boucheix \\ Université de Bourgogne, LEAD/CNRS, UMR 5022, Pôle AAFE, Esplanade Erasme, 21065 Dijon, BP: 26513 \\ E-mail: Jean-Michel.Boucheix@u-bourgogne.fr.
}

Michèle Coiron

C.H.U, Cadre-Infirmière, Responsable de Formation.

\begin{abstract}
This study assesses the ergonomic quality of a new writing format used for the written transmission of activity during a nurse's handover in a hospital. This format called "targeted or focussed transmission" comes from a new prescription of hospital management designed to improve the written handover process. Our research focused on the information filtering process for each patient concerned by a shift handover. A three step methodology was designed, with the participation of 9 nurses in charge of 70 patients: (1) nursing work analysis before the handover, (2) oral handover analysis, (3) written handover analysis. Results show that the new writing format does not match the nurses' needs to perform high quality handovers. The main clues, clinical signs and patient behaviour, used by nurses during an oral handover, to supervise the monitoring of the patient state disappear from the content of the written handover document. Regulation activities carried out to manage areas of dysfunction within the patient's state, which are a main topic in the oral handover, are not included in the written handover document. The different roles played by oral dialogue and written traces in the patient monitoring activity have been analysed and discussed. Finally, a cognitive model of professional written production is proposed and discussed and also some recommendations to design writing tools for the optimization of written handover documents.
\end{abstract}

KEYWORDS

Working handover, Written transmissions, Written production model.

\section{1.- Introduction}

À travers une analyse de l'activité de relèves de poste entre infirmières, le but de ce travail est d'évaluer l'ergonomie d'un nouveau format d'écriture des transmissions écrites conçu et prescrit par l'institution hospitalière. Dans un contexte d'optimisation de la « qualité » du suivi des malades, de nombreuses structures hospitalières ont souhaité satisfaire aux critères d'accréditation des établissements de soin. Cette démarche prescrivait la mise en œuvre d'indicateurs de traçabilité du travail. Ceux-ci sont associés à une exigence "d'efficience accrue », dans un contexte de diminution des coûts d'hospitalisation et d'augmentation des entrées et sorties de courtes durées liées à l'utilisation croissante de technologie d'explorations médicales. Bref, la charge informative croît, alors que les données contextuelles sur les malades diminuent. Dans ce cadre, le CHU (d'une ville de 250000 habitants) a conçu et développé, en partenariat avec le personnel cadre infirmier, une nouvelle structure textuelle formelle de transmission écrite du travail de l'infirmière lors des relèves de poste. Les 
transmissions dites « ciblées » ont pour but de focaliser l'écrit, concernant chaque patient, sur une cible, c'est-à-dire sur une information saillante de la dernière relève du poste, puis de développer les informations concernant cette cible selon une structure formelle composée de trois critères : les Données, les Actions réalisées et les Résultats obtenus (D.A.R., cf. tableau 1 et ci-dessous). La finalité de cette nouvelle prescription était de permettre un meilleur suivi, en temps réel et précis, de l'état du malade en sélectionnant les aspects significatifs de l'évolution du patient (système dynamique, Cellier, de Keyser, \& Valot, 1996 ; Hoc, 1996). De cette manière, pourrait s'effectuer un contrôle « on line » des transformations quotidiennes de l'état du malade, visibles, traçables, diminuant ainsi d'autant les éventuels risques d'accidents post-opératoires.

La conception et la mise en œuvre de ce nouvel " outil d'écriture », utilisé au CHU ici concerné, ont été réalisées avant notre intervention, sans qu'aucune analyse préalable de l'activité habituelle des écrits spontanés existants des infirmières n'ait été effectuée. Or, les infirmières ont une longue pratique de la transmission et l'on peut se poser la question de l'adaptation de ce nouveau format aux besoins réels des infirmières. Pour l'institution, le motif de la mise en place de ce nouveau format apparaissait double: disposer de documents permettant une traçabilité du travail infirmier et concevoir une aide à la rédaction de relèves écrites précises. Le format de transmission proposé est-il adapté à ces objectifs? Devant les difficultés rapportées par les professionnelles à la suite de la mise en œuvre de cette nouvelle prescription, la demande initiale de l'institution hospitalière à notre égard avait été de construire une formation professionnelle visant à développer les compétences des infirmières à la rédaction de ce format spécifique de transmissions écrites dites « ciblées ».

Avant d'envisager toute action de formation, il nous est apparu nécessaire, au plan ergonomique, de vérifier l'adéquation de cet outil de rédaction avec l'activité de transmission des infirmières lors des relèves (de Montmollin, 1986 ; Rogalski, \& Samurçay, 1994 ; Leplat, 1997, Pastré, 1997 ; Boucheix, 2003). L'objectif du travail présenté dans cet article est d'évaluer l'adaptation ergonomique de ce nouveau format de rédaction des transmissions écrites prescrit par l'hôpital aux besoins de professionnelles lors de la relève de poste. Pour cela, nous avons conduit une analyse de l'activité de transmission orale et écrite lors de la relève de poste chez 9 infirmières en charge de 70 patients. Cette analyse était précédée d'une étude de la période d'activité auprès de ces patients précédant la relève. Nous nous intéressons particulièrement à la dimension du filtrage de l'information. Nous formulerons, à la fin de notre étude, des préconisations pour l'optimisation de ces écrits.

\section{1.- Qu'est-ce que la transmission ciblée, D.A.R? Analyse de la prescription}

Notre but est l'évaluation de l'usage des écrits ciblés, mais qu'est-ce qu'une transmission écrite dite ciblée? Dans cette partie nous présenterons d'abord les bénéfices qui étaient attendus a priori par l'hôpital de la mise œuvre des transmissions ciblées et une analyse des contraintes inhérentes à cette nouvelle prescription.

D'abord, la transmission ciblée (voir l'exemple présenté tableau 1) impliquerait un tri de l'information concernant l'évolution du patient. Ce tri nécessite de choisir une cible qui correspond à une information nouvelle saillante concernant le patient et de développer les informations cliniques la concernant. Cette cible est choisie par l'infirmière. Autour d'un thème principal, appelé cible, les informations décrivant celle-ci doivent être classées selon trois critères: les Données, les Actions, les Résultats: D.A.R. Un exemple de transformation d'une transmission « habituelle » (issue de notre échantillon) en une transmission ciblée est présenté dans le tableau 1. 
Transmission antérieure à la transmission ciblée

\begin{tabular}{|c|c|c|c|c|}
\hline Date & Nom & \multirow{2}{*}{\multicolumn{3}{|c|}{ A souffert en début de nuit }} \\
\hline $6 / 04 / 98$ & $X$ & & & \\
\hline \multicolumn{5}{|c|}{ Transmission ciblée } \\
\hline Date & Nom & Cible & DAR & \\
\hline \multirow[t]{2}{*}{$20 \mathrm{H}$} & $\mathrm{X}$ & Douleur & D & $\begin{array}{l}\text { Douleur abdminale intense, position antalgique } \\
\text { en chien de fusil, gémissement }\end{array}$ \\
\hline & & & $\mathbf{A}$ & Injection Prodalfon et Spasfon \\
\hline $21 \mathrm{H}$ & & & $\mathbf{R}$ & Patient calmé, ne se plaint plus \\
\hline
\end{tabular}

Tableau 1: Exemple de transmission ciblée sous la forme D.A.R. (Données, Actions, Résultats)

Selon la prescription, le critère de choix de la cible correspond à la « survenue d'un événement physiologique ou psychologique d'un état ou d'une sensation qui n'étaient pas présents et qui modifient la situation du patient $»$. Ce choix apparait faussement simple. Il implique un tri dans les informations mémorisées issues du travail. Deux types de critères déterminent cette sélection. Le premier concerne la saillance, c'est-à-dire la pertinence et la nouveauté de l'information apportée par rapport à la pathologie et au pronostic prévu de l'évolution de la santé du patient. Une fois le choix réalisé sur cette dimension, l'infirmière doit passer de son mode d'écriture habituel des informations, c'est-à-dire suivant un ordre conforme à celui de la récupération spontanée des informations en mémoire (chronologique, comme dans l'exemple du tableau 1: «A souffert en début de nuit, calmé par Prodaf... »), à un mode d'organisation des informations écrites suivant un classement de cellesci en fonction d'une nomenclature ou catégorisation nouvelle. Le développement des informations concernant la cible choisie doit être réalisé selon la structure formelle en DAR. D'un point de vue opératif, il n'est pas certain que ce nouveau format soit réellement plus fonctionnel que celui utilisé spontanément par les infirmières. S'ajoute également une norme d'acceptabilité « institutionnelle » de ce que l'on peut écrire par rapport à ce que l'on peut énoncer lors de la transmission orale.

La transmission ciblée aurait également pour but d'améliorer la précision des informations cliniques. Dans l'exemple ci-dessus, l'information « a souffert toute la nuit » se développerait en précision de la manière suivante « douleur abdominale, position antalgique en chien de fusil, pousse de gémissements ». Ces précisions pourraient fournir des indices susceptibles d'optimiser le diagnostic et l'anticipation des événements futurs. Ces diagnostics dits « infirmiers » correspondent à un jugement clinique (diagnostic et pronostic) sur l'évolution du patient, servant de base (comme complément de la prescription médicale) au choix des interventions de soins de l'infirmière. Celle-ci analyse les réactions du patient à la prescription du médecin, prévient les comportements à risque, régule l'état du patient (confort, bien être). Cette activité, sur laquelle s'appuie l'élaboration de la transmission écrite, repose sur le repérage de "signes cliniques », la production de raisonnements (mise en relation des signes avec les connaissances préalables, suivie d'hypothèses). Des décisions d'action peuvent ensuite être envisagées. Un exemple d'évolution possible d'un diagnostic de ce type à partir des manifestations cliniques est donné dans l'exemple suivant:

Diagnostic actuel, à partir de signes:

«Anxiété reliée à l'hospitalisation se manifestant par un tremblement de la voix »;

Diagnostic potentiel, à partir de risques:

« Risque infectieux lié à une altération du système immunitaire ».

Notons cependant que, dans l'exemple du tableau 1, la formulation spontanée «A souffert en début de nuit, calmé par Prodaf, a peu dormi » contient aussi information plutôt centrée sur le vécu du patient et la relation avec ce dernier, alors que la transmission ciblée relève davantage du diagnostic technique «Douleur abdominale intense, position antalgique en chien de fusil, gémissements Injection Prodalfan et Spasfon. Patient calmé, ne se plaint plus ». Or, la relation et le contact avec le 
patient constituent des aspects fondamentaux du métier de soin.

Dans la transmission ciblée, il s'agirait aussi d'éviter les répétitions. Le ciblage des informations permettrait de diminuer la redondance importante des transmissions d'une relève à l'autre. Cependant, notons que parfois, cette redondance pourrait avoir une fonctionnalité. Une présentation standardisée permettrait de retrouver facilement les informations. La distribution des informations développées selon la structure formelle DAR devrait optimiser le classement des informations et faciliter leur récupération, à condition que les infirmières s'approprient l'outil.

Enfin, du fait de l'objectif de traçabilité qui est recherché par l'institution, le DAR pourrait induire chez l'infirmière une activité d'évaluation de « l'acceptabilité » des informations développées. En effet, la trace écrite revêt un caractère permanent accessible à plusieurs catégories de destinataires. Cet écrit induit ainsi des normes d'acceptabilité concernant la nature des informations retenues, qui peuvent être différentes de celles des relèves orales.

Bref, la rédaction de transmissions ciblées impliquerait les contraintes suivantes: filtrer et trier les informations, améliorer le contenu des descriptions cliniques, intégrer des diagnostics infirmiers, éviter les répétitions d'une relève à l'autre, et retrouver plus facilement les informations que dans des écrits « tout venant ». En ce sens, ils constitueraient des séries de descriptions sur l'évolution de l'état d'un organisme comparable à un « système dynamique » (Hoc, 1996). Mais cette formalisation est-elle adaptée à l'activité de l'infirmière?

\section{2.- Les difficultés rencontrées dans la mise en ouvre de la transmission ciblée, DAR.}

L'objectif prescrit par l'institution lors de l'introduction de transmissions écrites était de « capitaliser des informations écrites fiables et pratiques pour assurer des soins continus » (texte prescriptif du processus de l'accréditation). Le but de cette nouvelle prescription était l'opérationnalisation du suivi par l'infirmière du système dynamique que constitue le malade, en relation avec le médecin. Mais, l'infirmière, en particulier expérimentée, met en œuvre depuis longtemps déjà des compétences de suivi opérationnel et de transmission à travers des supports d'informations écrits (feuilles de soins, fiches de suivi, relève écrite habituelle) et aussi oraux (relève orale) (cf. Errard, Kostulski, \& Trognon, 1996). Les infirmières exercent déjà un tri des informations dans leurs écrits habituels, comme le montre l'exemple du tableau 1 . Au cours de ces activités se développent les compétences spécifiques $\mathrm{du}$ « diagnostic infirmier $»$.

Les travaux consacrés au travail hospitalier et notamment à la relève orale ont montré que les professionnels développaient efficacement une véritable intelligence, une véritable compétence collective (Grosjean \& Lacoste, 1999; Cosnier, Grosjean, \& Lacoste, 1993). Par ailleurs, la relève ne se réduit pas seulement à une transmission unilatérale d'informations, il s'agit d'une interaction constructive entre les interlocuteurs au cours de laquelle des problèmes sont analysés et résolus (Grusenmeyer, 1995, 1996; Grusenmeyer \& Trognon, 1996) comme dans les interactions des situations de service (Cerf, Valléry, \& Boucheix, 2004 ; Boucheix, 2005; Cerf, \& Falzon, 2005; Valléry, Leduc, Boucheix, \& Cerf, 2005). Grosjean et Lacoste (1999) ont montré qu'écrit et oral constituaient deux modalités complémentaires de suivi du patient, servant des fonctionnalités différentes.

De leur côté, les travaux de psychologie cognitive de la production du langage ont également montré que production (ou interaction) orale et production écrite constituaient deux modalités de travail. Les contraintes fonctionnelles de l'une et de l'autre modalités sont distinctes (Bourdin, 1994; Heurley, 1994 ; Fayol, 1997; Alamargot, \& Dansac, 2003). Dans un cas, le destinataire est présent et simultanément actif dans le cadre d'une communication multimodale accompagnée de nombreux feed-back permettant la construction d'un référentiel commun (Giboin, 2004), dans l'autre le destinataire est absent. La transmission ciblée requiert, pour le scripteur, la gestion des contraintes de deux ordres. Du point de vue de la rédaction, il s'agit de passer d'une écriture spontanée habituelle des connaissances issues directement de l'action qui correspond à une stratégie de récupération en mémoire de 
connaissances au fur et à mesure de l'écriture (Fayol, 1997) à une présentation des informations organisées autour de thématiques choisies (parmi d'autres possibles) et selon une organisation textuelle prescrite. Cette seconde méthode implique la mise en œuvre d'une stratégie de «transformation » des connaissances (Levelt, 1989 ; Levelt, \& Maassen, 1991; Hayes, \& Nash, 1996; Fayol, 1997), en fonction d'un but et d'un destinataire. Bourdin (1994) a montré que ce type d'activité de transformation était particulièrement difficile. De plus, s'ajoutent de contraintes d'ordre socio-institutionnelles dans la rédaction des écrits de travail (Boutet, 1995): type de destinataire, utilisation et statut de l'écrit, évaluation sous-jacente de l'activité des professionnels à travers les écrits. Dans tous les cas, il s'agit d'un langage opératif (Falzon, 1989), mais il est écrit et l'on connaît peu de chose sur le langage opératif écrit.

Cette nouvelle prescription d'écriture s'adresse à l'ensemble des infirmières du CHU. Lors de sa conception, qui précédait la demande qui nous a été formulée, aucune analyse préalable de l'activité des professionnels ni de l'acceptabilité de nouvel outil n'avait été réalisée. Seule une information avait été donnée. Avant la généralisation prévue à l'ensemble du CHU, plusieurs services étudiés ici avaient déjà adopté la pratique des transmissions ciblées de type DAR. Lors de nos entretiens exploratoires, les infirmières concernées ont fait état de difficultés dans la mise en œuvre spontanée de l'écriture de transmissions ciblées, alors même qu'elles déclaraient une volonté d'améliorer ces transmissions.

Nous avons commencé par conduire une première phase d'observation exploratoire directe auprès des infirmières utilisant la démarche de transmissions ciblées (entretiens directs concernant des écrits et observations en situations) afin de confirmer la réalité du problème. Nous cherchions ainsi à mieux connaître la définition et les représentations que les infirmières concevaient de la notion de cible. Nous avons pu relever les difficultés déclarées suivantes:

- Même si l'objectif de la relève écrite n'est pas de se substituer à la relève orale mais de la compléter, il existerait une perte d'information trop importante entre la relève orale et la relève écrite, qui pose le problème de la sélection.

- Le choix de la cible (catégorisation) et la formulation (en DAR) posent souvent un problème. Cette difficulté a pour effet le repli vers des cibles « génériques » issues non pas de l'identification de signes cliniques mais de l'utilisation de nomenclatures génériques classiques dans le travail infirmier mais peu informatives sur l'évolution de l'état du malade. Par exemple: « alimentation », « respiration».

- La recherche d'informations précises, qui ne sont pas systématiquement accessibles au moment de la rédaction, peut générer des biais concernant la réalité des données relatées à propos du patient.

- L'organisation des informations ne suit pas la structure DAR. Par exemple, le chiffre d'une prise de la tension peut être classé en donnée ou résultat, pour une même cible: « risque d'hypertension ».

- Il est difficile de différencier une action à envisager d'une action déjà réalisée. Par exemple: « doit rester à jeun » et « humidification arrêtée ».

- Les résultats des actions entreprises par l'infirmière, qui sont les véritables marqueurs du suivi de l'évolution des problèmes rencontrés, sont souvent absents de l'écrit.

- Il existe une grande variabilité à la fois interindividuelle et intra-individuelle (qualitative et quantitative) dans la précision des informations rapportées.

- Bref, l'expression du suivi infirmier à travers la transmission ciblée apparaissait difficile. L'objectif de ce travail est d'évaluer l'adaptation de la transmission ciblée à l'activité de relève chez les infirmières. Dans ce but, nous avons réalisé une analyse de l'activité de transmission écrite chez une population d'infirmières en activité utilisant ce nouveau système de notation, en relation avec leur activité de supervision de la transformation de l'état des malades. Nous nous sommes centrés particulièrement sur le processus de filtrage de l'information pertinente 
concernant l'évolution du malade.

- La transmission ciblée est-elle mise en œuvre et comment?

- Dans quelle mesure ce format d'écrit contient et conserve une trace de l'activité réelle de suivi de l'infirmière (diagnostics, pronostics, risques, incidents, régulations)?

- Inversement, quelles informations issues de l'activité de l'infirmière ne figurent pas dans les transmissions?

- Quels sont les processus cognitifs mis en œuvre pendant l'écriture de la transmission? Quelle est la fonction de cette transmission ciblée comparativement à la relève orale?

De la réponse à ces questions dépend une orientation vers des préconisations potentiellement très différentes.

\section{2.- Méthode}

Compte tenu des difficultés évoquées précédemment, quelles traces significatives de l'activité de l'infirmière les transmissions ciblées comporteront-elles? Et, en particulier conserveront-elles des traces du diagnostic infirmier qui se révèle crucial concernant le suivi du malade? Le test de cette hypothèse nécessite que l'on puisse comparer le contenu et la forme des écrits au contenu et à la forme des autres activités de l'infirmière concernant les malades ayant fait l'objet de ces écrits: en particulier le contenu des relèves orales et le contenu des connaissances et des représentations de l'infirmière pour chaque malade présent et issus de l'activité qui a précédé la relève. De cette comparaison, il est possible de déduire à la fois l'existence de traces (écrites et nommées) de l'activité de l'infirmière et la nature de buts de suivi réellement mis en œuvre pour la réalisation de cet écrit professionnel. En effet, au moment de la rédaction de la transmission selon le nouveau format, l'infirmière doit se livrer à un « calcul» pour choisir puis transformer et mettre en mots les produits d'une sélection d'information, ce « calcul» dépend des représentations mémorisées par l'infirmière au cours de son activité et pendant la relève orale (cette dernière étant en général effectuée préalablement), des contraintes liées aux destinataires potentiels de cet écrit, et des contraintes propres à l'activité rédactionnelle.

\section{1.- Population}

Nous avons sollicité 9 infirmières réalisant quotidiennement des transmissions écrites ciblées, en prenant en compte une variable pouvant se révéler sensible à cette activité de transmission qui est l'expertise. La répartition est la suivante: trois expertes reconnues par leurs pairs pour la qualité de leurs transmissions (ayant au moins 10 années d'expériences dans différents services), quatre novices (ayant entre 1 et moins de trois années d'expérience dans différents services) et deux professionnelles ayant un statut « intermédiaire » (plus de 3 années d'expériences dans différents services) dites expérimentées. Trois services différents ont été sollicités, (médecine, neurologie et néphrologie) qui sont comparables en ce qui concerne la gravité des pathologies rencontrées (opérations).

\section{2.- Démarche et procédure}

Nous avons suivi une démarche en trois phases chronologiques présentée dans le tableau 2. Cette démarche est fondée sur la mise en relation (comparaison) de l'activité réelle de chaque infirmière descendante (avec chaque malade), observée juste avant les transmissions orales et écrites de la relève de poste pour les mêmes malades, au cours du cycle de travail. Les infirmières montantes, bénéficiaires de cette relève, avaient été absentes depuis moins d'un jour à trois jours. 


\begin{tabular}{|c|c|c|c|c|}
\hline Déroulement & Phases & Objectifs & Processus étudié & $\begin{array}{l}\text { Résultat } \\
\text { Filtrage }\end{array}$ \\
\hline & $\begin{array}{l}\text { Phase1- Analyse } \\
\text { de l'activité } \\
\text { préalable à la } \\
\text { transmission } \\
\text { écrite. } \\
\text { (70 patients, } 9 \\
\text { infirmières) }\end{array}$ & $\begin{array}{l}\text { Représentation } \\
\text { référentielles } \\
\text { (RR) individuelles } \\
\text { préalables } \\
\text { mémorisés pour } \\
\text { chaque malade }\end{array}$ & \multirow[t]{3}{*}{$\mid$} & $\begin{array}{l}\text { Connaissances } \\
\text { et } \\
\text { représentations } \\
\text { de référence } \\
\text { (RR) }\end{array}$ \\
\hline & $\begin{array}{c}\text { Phase 2- Analyse } \\
\text { des transmissions } \\
\text { orales }\end{array}$ & $\begin{array}{c}\text { Informations } \\
\text { transmises lors } \\
\text { de la relève orale } \\
\text { (RO) }\end{array}$ & & $\begin{array}{l}\text { Comparaison } \\
\text { CR et RO }\end{array}$ \\
\hline$\downarrow$ & $\begin{array}{l}\text { Phase 3- Analyse } \\
\text { des transmissions } \\
\text { écrites }\end{array}$ & $\begin{array}{l}\text { Production écrite } \\
\text { (PE) Informations } \\
\text { et processus de } \\
\text { transcription }\end{array}$ & & $\begin{array}{l}\text { Comparaison } \\
\text { CR, RO et PE }\end{array}$ \\
\hline
\end{tabular}

Tableau 2: Méthodologie et chronologie de l'analyse de l'activité de transmission écrite

La première phase (tableau 2) avait pour but de constituer une base de connaissances et de représentations référentielles concernant le contenu du suivi de chaque malade pour chaque infirmière. Cette référence reflétait les représentations préalables mémorisées pour chaque malade dont chaque infirmière disposait au moment de l'élaboration de la transmission orale puis de la transmission écrite. Cette Phase 1 a été réalisée avec l'analyse de l'activité préalable. Il s'agissait d'observations directes et d'enregistrements en situation: nous avons réalisé des observations directes des actions, comportements et interactions de soin de chaque infirmière effectuées en situation et accompagnées d'enregistrements audio (micros sans fil) de chaque interaction (avec le patient ou un collègue) pour chaque infirmière (pour un total de 81 heures). Ont été relevées toutes les actions de chaque infirmière, la relève précédant le cycle d'activité, les interactions avec chaque patient, la relève suivant le cycle d'activité, orale et écrite. Ces observations et enregistrements ont ensuite été intégralement retranscrits. Ont été précisément relevées, les catégories de soins avec le but de l'infirmière pour chaque action: distribution et prise de médicament, prise et mesure de tension, demandes d'informations, soins quotidiens (pansements), et le contenu des informations échangées avec le patient.

Suivait un entretien-rappel individuel des représentations mémorisées qui était conduit juste après les observations directes. Cette épreuve avait pour but de transcrire le plus exhaustivement possible les représentations et connaissances mémorisées par l'infirmière concernant la pathologie et l'évolution de chaque malade. Il était demandé aux professionnelles de faire la distinction entre, d'une part les représentations antérieures au jour de l'observation et d'autre part, celles issues de la période d'observation. Cet entretien-rappel avait lieu le surlendemain de l'observation. Afin de faciliter la récupération des représentations pour chaque malade, l'infirmière disposait des documents concernant chaque patient (fiches de soins, et prescription) et des traces écrites des enregistrements réalisés pendant l'observation qui avait précédé.

Bien que les données relevées constituaient un riche matériau pour une analyse détaillée, l'exploitation des données de cette phase ne correspondait pas à une analyse complète l'activité précédant la relève, mais plutôt à un support qui permettait ensuite de recueillir rigoureusement les représentations des infirmières concernant les patients. Cette réduction convenait mieux aux objectifs limités de comparaison de cette étude. 
La deuxième phase avait pour but l'analyse des transmissions orales pour chaque infirmière observée concernant les 70 patients dont les professionnelles avaient la charge durant l'activité précédente (phase 1). La répartition de ces malades était la suivante: 23 pour le groupe des novices, 22 pour le groupe des expérimentées et 30 pour les expertes. Soixante-dix relèves ont été enregistrées, pour l'ensemble des patients et des infirmières de l'échantillon. Les énoncés issus des retranscriptions intégrales ont ensuite été analysés.

La troisième phase portait sur les transmissions écrites. Elle avait pour objectif d'analyser le contenu et le processus de production des transmissions, pour le mêmes 70 patients, par les neuf infirmières. Nous nous sommes intéressés en particulier aux traces de l'activité de suivi de l'infirmière pour chaque malade, et aux mécanismes de transcription de ces traces dans le cadre de la structure formelle du DAR. Soixante-dix relèves écrites ont été recueillies, transcrites intégralement puis analysées, correspondant aux mêmes malades que les observations et les transmissions orales. Ensuite, dans le but de recueillir des informations concernant les règles d'écriture et le processus de production des écrits, nous avons conduit, pour chaque écrit, le lendemain de la transmission et pour chaque infirmière, un entretien d'explicitation par l'infirmière portant sur chacune des transmissions qu'elle avait écrite. Cette technique de type protocole verbal, adaptée de Bisseret, Sébillotte et Falzon (1999), était menée avec le support écrit sous les yeux de l'infirmière pour chaque transmission et durait environ deux heures par professionnelle. Les critères d'analyse des données issues des techniques utilisées pour chaque phase sont exposés ensuite dans la présentation des résultats.

Enfin (dernière colonne du tableau 2), les données issues de trois phases de notre démarche ont été mises en relation. Le but de cette comparaison était de préciser le processus de « filtrage » de l'information, pour la même infirmière, en partant des représentations référentielles (RR), à la transmission orale (RO), et finalement écrite (PE). Bien évidemment, les échanges d'informations et les interactions ne peuvent se réduire à ce processus de filtrage formulé sous la forme "d'équation » dans le tableau 2. Cependant, l'étude de ce processus de filtrage constitue une dimension importante pour examiner l'information finalement sélectionnée et retenue dans la transmission écrite.

\section{3.- Résultats}

\section{1.- Phase 1- L'analyse des observations : les représentations référentielles préalables}

Le but de ce travail était centré sur le processus des productions écrites. En conséquence, notre objectif dans la description des résultats de cette première phase n'a pas été de présenter en détail les données issues des 81 heures d'observations de chaque professionnelle. Il s'agissait de constituer une base de représentations référentielles pour chaque malade permettant de mieux comprendre la nature et le filtrage des informations présentes dans les transmissions écrites, qui seront analysées en détail phase 3 . Nous nous limiterons donc pour la présentation des résultats de cette première phase, à un exposé des activités effectuées d'une part, de l'ampleur (quantitative) des connaissances mémorisées d'autre part, et enfin, des thématiques et sources principales des représentations référentielles concernant chaque patient, constituant les représentations des situations préalables à la relève des professionnelles.

Nous évoquerons brièvement les résultats des observations puis ceux de l'épreuve d'entretien-rappel des connaissances.

Les activités observées étaient les suivantes :

- les soins en séries prévus, en fonction d'un horaire précis: prise de sang, de tension, de médicaments, injections;

- les activités de soins individualisés, liées à l'évolution de l'état du malade: aide à la toilette, préparation pré-opératoire, pansements, confort; 
- des activités concernant des soins de régulation imprévues: changement d'état du malade, résultats d'examens;

- les interactions verbales avec chaque patient qui sont réalisées en concomitance avec les activités de soins précédemment évoquées et sont plutôt centrées sur le vécu et la relation avec le malade;

- les interactions avec les collègues et les actions réalisées en dehors de la chambre des patients (fiches à compléter, par exemple);

- Les activités de transmission des informations, orales et écrites, lors des relèves de poste. Ces dernières duraient au minimum 30 minutes par relève. Les transmissions orales étaient généralement collectives, les transmissions écrites individuelles. La transmission écrite a été réalisée pour chaque malade avant la relève orale pour 7 infirmières sur 9 , en cours de travail pour 1 infirmière et après la relève orale pour 1 . Nous avons également observé de grandes variations dans l'ordre d'écriture des informations: certaines infirmières commençaient par les cibles avant le développement de celle-ci, d'autres commençaient par les informations et écrivaient ensuite la cible. Certaines infirmières lisaient la transmission écrite précédente d'autres, majoritaires, ne le faisaient pas.

Ainsi, les représentations mémorisées par les infirmières étaient issues des sources d'activité suivantes:

- les activités observées relevées ci-dessus;

- les prises d'informations verbales et non verbales enregistrées: visuelles et proprioceptives;

- les actions réalisées qui le plus souvent étaient issues de la prescription médicale;

- les actions de réajustements et de régulation d'un dysfonctionnement chez le malade.

L'ampleur des prises d'informations des professionnelles, à travers la fréquence des interactions (verbales ou non) avec le patient, qui est un (des) indicateur(s) relié à la quantité de connaissances préalables mémorisées pour chaque malade, diffère fortement en fonction du niveau expertise. En effet, le nombre moyen d'interactions par malade est significativement plus élevé chez les expertes ( 7,9 pour 238 interactions avec 30 malades) que chez les novices (5,5 pour 127 interactions avec 23 malades) ou les expérimentées $(4,6$ pour 102 interactions avec 22 malades $)\left(\mathrm{Chi}^{2}=20.91, p<.01\right)$. Les taux d'interactions étant plus élevés chez les experts, nous pouvons supposer que les connaissances préalables mémorisées sont plus nombreuses et/ou plus précises chez ces professionnelles. Bien sûr la quantité de connaissances (et des interactions) n'est pas nécessairement indicative de leur pertinence, ni de leur fonction ou de leur contenu. Mais les travaux d'ergonomie cognitive concernant le diagnostic en médecine montrent que cet indicateur de quantité est de grande utilité. Quantité et surtout flexibilité des représentations opératives constituent des dimensions critiques de la compétence en médecine et en soins (Raufaste, 2001).

Quelles sont les représentations référentielles issues de l'épreuve d'entretien-rappel? Les données verbales de cette épreuve pour chaque infirmière ont été retranscrites. Nous avons ensuite catégorisé chaque énoncé (proposition ou groupe de propositions reliées à un thème), en fonction du type de source des informations concernées. Ces sources (S) sont au nombre de 7:

- S1- Les interactions verbales avec le patient (ex: «...pour les soins de 14h. Je lui ai dit bonjour, il n'a pas bien répondu... c'est une façon d'évaluer leur conscience... pour quelqu'un qui devait aller mieux, je trouvais qu'il répondait pas beaucoup »);

- S2- L'observation des signes cliniques et du comportement durant les interactions verbales ou non verbales (ex: " quand je l'emmenais à la balance, je sentais qu'il ne voulait pas pousser le pied à sérum, ça le gêne »);

- S3- Les informations nouvelles de la relève de poste (ex: " l'infirmière de nuit m'avait dit... qu'elle avait arraché sa perfusion »);

- S4- Les connaissances antérieures au jour de l'observation (ex: " elle a eu un problème de 
douleur thoracique,... elle avait eu un électro, un bilan cardiaque qui n'avait rien montré »);

- S5- L'exécution de la prescription médicale (ex: " j'avais la surveillance après l'artériographie... tension normale, pouls étaient bons »);

- S6- La pathologie intrinsèque du patient (ex: " elle était présentée comme éthylique avec 3 grammes... »);

- S7- Les actions autonomes (ex: «Par rapport à ses instillations, je lui ai fait faire un bain de bouche »).

Cette catégorisation servira de base de référence à l'analyse des informations contenues dans les transmissions orales puis écrites.

\section{2.- Phase 2- Les transmissions orales}

Quel est le contenu dans les transmissions orales? C'est la question à laquelle les analyses conduites sur le corpus de 70 transmissions orales ont tenté de répondre. Pour ce faire, nous avons découpé chaque relève en unités plus petites: le tour de parole, l'énoncé, et la séquence. Le tour de parole est un indicateur général de la densité de la transmission. Nous avons relevé 617 tours de paroles pour l'ensemble des malades, soit un taux moyen de 8,1 tours de parole par malade. L'énoncé correspond à une unité d'information complète sur un thème, proche de la proposition. La séquence se compose d'un ensemble d'énoncés cohérents concernant le même thème. Elle constitue un bon indicateur du but poursuivi dans les thèmes abordés à travers les énoncés.

\subsection{1.- Les thèmes abordés dans les énoncés lors des échanges}

Nous avons relevé 1184 énoncés, pour 70 transmissions soit une moyenne de 17 énoncés environ par transmission. Nous avons classé chaque énoncé en fonction de son objet, c'est-à-dire du thème de l'information abordé concernant le malade. Les résultats quantitatifs de ce classement sont consignés dans le tableau 3, ainsi qu'un exemple de la catégorisation effectuée.

\begin{tabular}{|c|c|c|}
\hline $\begin{array}{l}\text { Thème de } \\
\text { référence }\end{array}$ & $\%$ & Exemple de catégorisation* \\
\hline $\begin{array}{l}\text { Prescription } \\
\text { médicale }\end{array}$ & 34 & \multirow{9}{*}{$\begin{array}{l}\text { « En face, Mme S (identité**) } \\
\text { qui a été opérée d'un hématome } \\
\text { sous-dural chronique (diagnostic } \\
\text { médical) qui ma foi n'est pas trop } \\
\text { mal (signe clinique global) euh.. elle } \\
\text { est encore perfusée (prescription) } \\
\text { et si elle mange bien il n’y aura } \\
\text { pas de problème à la faire manger } \\
\text { (comportement) elle a un déficit } \\
\text { neuro (signe clinique), juste c'est } \\
\text { une dame qui est insulino-dépendante } \\
\text { (pathologie) qui a un dextro toutes } \\
\text { les } 4 \text { heures (prescription)» }\end{array}$} \\
\hline Comportement & 18 & \\
\hline Signes cliniques & 16 & \\
\hline Diagnostic & 9 & \\
\hline Motif & 6 & \\
\hline Identité & 4 & \\
\hline Pathologies & 4 & \\
\hline Organisation générale & 4 & \\
\hline Divers & 4 & \\
\hline
\end{tabular}

* Nous avons choisi un exemple simple et compréhensible. Dans beaucoup de cas les termes techniques nombreux et spécialisés rendent la compréhension difficile pour un non professionnel.

** L'identité correspond simplement à l'énoncé du nom du patient et n'est relevée qu'une fois (par transmission).

Tableau 3 : Répartition (en \%) de différents thèmes de référence des énoncés des transmissions orales

Nous avons constaté la prédominance de trois types d'informations. Celles concernant la prescription médicale et sa mise en œuvre, celles portant sur les comportements, et celles ayant trait aux signes cliniques qui apparaissent. Ces deux dernières catégories, signes cliniques et comportements représentaient $34 \%$ des énoncés. Ils correspondaient aux véritables indicateurs de l'activité de su- 
pervision de l'infirmière et de sa gestion des risques. En effet, ces indicateurs (signes cliniques en particulier) concernent les modifications et les changements de l'état du malade, à partir d'une base de connaissances structurées par le diagnostic médical initial, la prescription et le motif de l'hospitalisation. Par ailleurs, ces indicateurs semblaient sensibles à l'expertise. Les infirmières expertes exprimaient plus souvent des énoncés liés à l'apparition de signes cliniques ou de comportements ( $40 \%$ ) que les infirmières novices ( $29 \%$ ) ou expérimentées (31\%). En revanche, les novices prenaient d'abord en compte la prescription médicale (40\%) en comparaison des expertes (32\%). Les autres types d'énoncés (identité du patient, motifs d'hospitalisation, organisation) se répartissaient également entre les niveaux d'expertise. Bref, l'étude des énoncés montre que la relève orale revêt une fonction d'échange concernant les représentations opératives des changements d'état (signes cliniques et comportements) et de leurs évolutions possibles chez le patient.

\subsection{2.- Les buts des infirmières dans les séquences d'échange}

Chaque séquence (247 au total) a été caractérisée en fonction de 3 critères. Le premier concernait la nature de la situation décrite, qui pouvait être normale (contenant par exemple l'énoncé de la prescription) ou relever d'un dysfonctionnement de l'état du patient (changement de l'état ou du comportement du patient). Le deuxième critère était relatif à l'objet abordé en 6 thèmes : prescription médicale, pathologie connue du patient, effets de la prescription, comportements, signes cliniques. Le troisième critère avait trait au niveau de régulation de la situation décrite. Quatre niveaux ont été définis : régulation réalisée, régulation à réaliser, échec de la régulation, absence d'une régulation. La régulation était dite réalisée quand l'infirmière indiquait qu'après action remédiatrice ou ajustement de la prescription, le malade avait recouvré son état antérieur à la régulation. Dans le cas d'une régulation à réaliser, l'échange de la relève contenait la solution pertinente à apporter au patient ainsi que la procédure à suivre, mais l'action correspondante n'avait pas encore été mise en œuvre au moment de la relève. L'échec de régulation correspondait à une situation où après dysfonctionnement de l'état du patient les différentes solutions de remédiation tentées avaient échoué au moment de la relève. Enfin, l'absence de régulation correspondait à une situation où des actions régulatrices à mettre en œuvre ne l'avaient pas été (ou n'avaient pas pu l'être). Voici deux exemples de ces situations : un dysfonctionnement à réguler - " elle a un cathlon sur la main. Enrubannée. À surveiller puisqu'a priori elle l'a arraché, elle a déjà enlevé les cathlons, elle était pas très présente, je dirais apathique "; une absence de régulation (ou échec): «doit boire, je l'ai pas trop fait ».

Sur les 247 séquences analysées, $49 \%$ portaient sur une situation normale, contre $51 \%$ qui concernaient un dysfonctionnement. Nous avons consigné dans la figure 1, la répartition des séquences par thème et en fonction du type de situation (normale ou non).

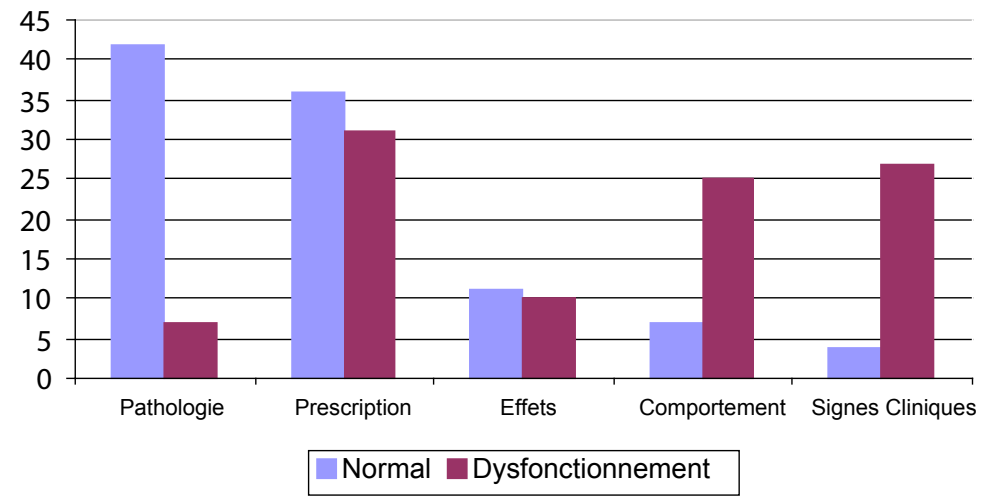

Figure 1 : Répartition (en \%) des séquences de la transmission orale par thèmes (Pathologie, Prescription médicale, Effets de la prescription, Comportements, Signes clinique) en fonction du type de situation (normale ou en dysfonctionnement).

Il est apparu que les taux de dysfonctionnement les plus élevés concernaient trois indicateurs: les prescriptions, les signes cliniques et les comportements. Alors qu'un dysfonctionnement concernant 
la prescription pouvait correspondre par exemple à un ajustement individualisé de la prescription au patient, les catégories signes cliniques et comportements étaient des indicateurs critiques propres aux situations de dysfonctionnement de l'état de santé du malade qui apparaissaient dans les transmissions orales. C'est la régulation de ces dysfonctionnements, pas nécessairement liés à la prescription, qui est au centre des échanges de la relève orale. La répartition des catégories de dysfonctionnements semblait différer selon le niveau d'expertise, comme le montre la figure 2.

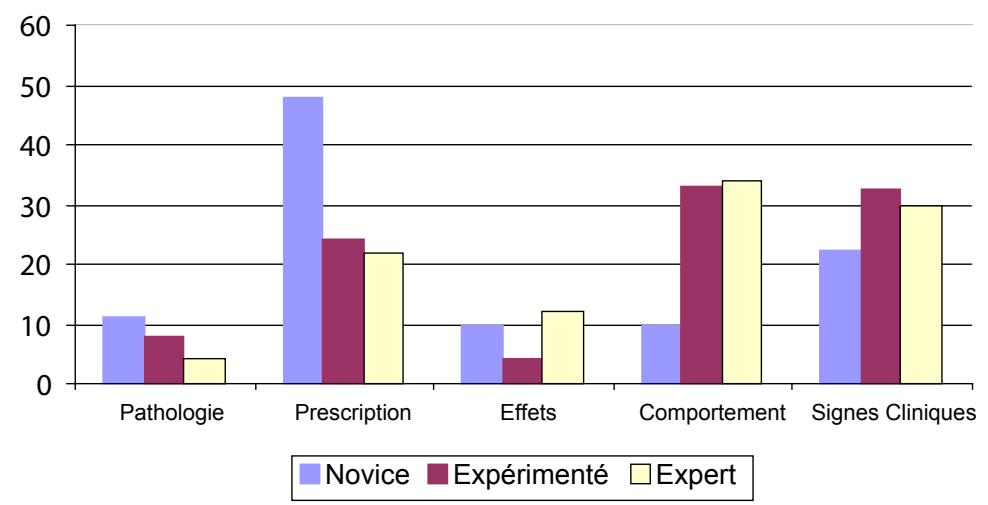

Figure 2 : Répartition des objets de dysfonctionnements selon le niveau d'expertise

Les experts, ainsi que les professionnelles expérimentées, échangeaient beaucoup plus d'informations liées aux signes cliniques et aux comportements que les novices, plus centrées sur la prescription. Or, les informations pertinentes pour la relève sont surtout les premières, qui constituent des traces de l'activité de suivi et de supervision du malade par l'infirmière. Remarquons que cette statistique indique une tendance puisque les données concernant les trois niveaux d'expertise ne concernent pas les mêmes situations de pathologie.

\subsection{3.- Les régulations comme enjeu des transmissions orales}

Quels sont les niveaux de régulation exprimés dans les transmissions orales? Nous avons indiqué ci-dessus quatre stades possibles de régulation. La répartition des séquences des transmissions orales comprenant l'évocation d'un dysfonctionnement, en fonction de ces quatre niveaux, est présentée tableau 4.

\begin{tabular}{|l|c|}
\hline \multicolumn{1}{|c|}{ Niveaux de la régulation } & Fréquences en \% \\
\hline Régulation réalisée & 49 \\
\hline Régulation à réaliser & 29 \\
\hline Echec de régulation & 12 \\
\hline Absence de régulation & 10 \\
\hline
\end{tabular}

Tableau 4 - Répartition des différents niveaux de régulation évoqués au cours de la relève orale

Il est ainsi apparu que $\mathbf{5 1 \%}$ des dysfonctionnements n'étaient pas encore régulés. Leur évocation dans la relève orale permet d'orienter l'activité de supervision de l'infirmière qui prend la relève. Enfin, les dysfonctionnements à réguler étaient plus souvent abordés par les expertes (40\% contre $15 \%$ pour les novices et $20 \%$ pour les expérimentées). L'expert semble anticiper les régulations des dysfonctionnements présents dans l'état du malade, surtout à partir des signes cliniques et comportementaux. Peuvent ainsi être discutés, durant la relève, au cours de l'interaction les modes de régulations à réaliser.

En résumé, l'analyse du contenu de la relève orale a permis de mettre en évidence sa fonction d'échange pragmatique concernant l'évolution de l'état du patient à partir d'indicateurs spécifiques aux professionnels concernant les signes cliniques et les variations du comportement. L'un des enjeux de la relève réside dans la gestion et la résolution des problèmes de dysfonctionnement et des 
régulations à réaliser. Il serait intéressant, mais en dehors des limites quantitatives d'un seul article, d'approfondir les types de dysfonctionnement régulés et non régulés, en les mettant par exemple en relation avec les données de l'analyse de l'activité préalable (étape 1).

Dans quelle mesure les informations pertinentes pour la supervision de l'état du malade, relevées dans les transmissions orales (signes cliniques, comportements, dysfonctionnements à réguler) étaient-elles présentes dans les transmissions ciblées, et de quelle manière? Quelles informations complémentaires à la relève orale, ou soustraits de celle-ci, contenaient les écrits ? Comment étaient produits et utilisés les écrits ciblés comparativement aux informations orales? C'est l'objet de l'analyse qui suit centrée sur les transmissions écrites.

\section{3.- Phase 3- Les transmissions écrites ciblées}

Nous avons analysé le corpus de 70 transmissions écrites. Une série d'exemples de ces transmissions écrites est fournie dans la figure 2. Dans un premier temps, nous présenterons les résultats des analyses des écrits eux-mêmes, puis dans un second temps nous nous intéresserons aux données issues des protocoles verbaux recueillies à propos de la rédaction de ces écrits.

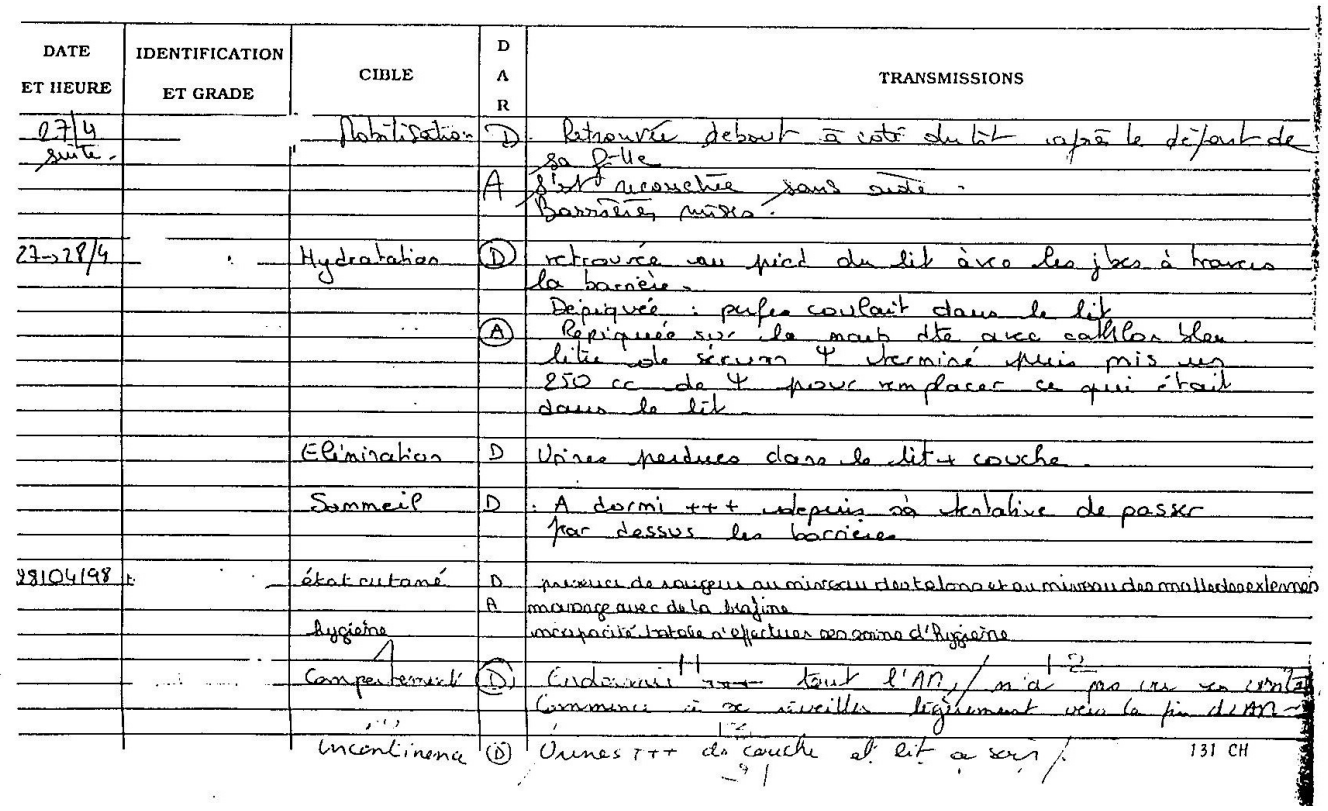

Figure 3 : Exemple de fiche de transmission écrite ciblée utilisée pour la relève

\subsection{1.- Le tri des informations contenues dans les transmissions écrites et l'usage de la formulation en DAR}

Nous avons étudié chaque transmission en fonction de la présence des éléments prescrits : présence de cible et tri des informations en DAR tout d'abord, analyse de la nature des cibles et de la structure des écrits, ensuite. Nous avons ainsi traité 115 cibles, tableau 5.

\begin{tabular}{|c|c|c|c|c|c|c|}
\hline Tri & Données & DA & Non classé & DAR & R & DARA/DRA \\
\hline$\%$ & 26 & 23 & 20 & 12 & 12 & 7 \\
\hline
\end{tabular}

Tableau 5 : Répartition (en \%) des cibles en fonction de leur structure (Données,

Résultats, Données + Actions, Données + Actions + Résultats, Non classé, Mixtes)

Nous avons observé que seulement $12 \%$ des transmissions suivaient les consignes d'écriture en DAR, $27 \%$ étaient classées selon des structures qui ne correspondaient pas aux consignes de la notation écrite, ce sont surtout les données qui prédominaient. Il n'était pas toujours aisé de distinguer les données des résultats. Dans notre classification, le résultat correspondait à une information 
explicite concernant l'effet (au cours du poste en cours) d'une action entreprise sur un paramètre (comportemental ou physiologique). Une donnée correspondait à une information précédant l'action. En résumé, à première vue, la structure DAR semble peu adaptée à l'activité de relève écrite actuelle des professionnelles, ou à tout le moins difficile à mettre en œuvre spontanément.

Pour approfondir ces résultats, nous avons procédé à un découpage en énoncés des développements des 115 cibles. Un énoncé correspondait à une proposition cohérente et/ou une expression plus petite qu'une proposition mais contenant une unité sémantique. Un énoncé correspondait donc à une unité fonctionnelle de l'écrit de la transmission. Par exemple, l'énoncé « on sait pas encore si elle doit être transfusée ou pas » comporte plusieurs (deux) propositions, alors que l'énoncé «incontinente » n'en constitue pas vraiment une. Mais ces deux énoncés expriment une unité fonctionnelle d'information médicale pour la transmission. En utilisant ce découpage, nous avons relevé 354 énoncés pour les 115 cibles trouvées dans les 70 transmissions écrites (correspondant aux situations observées durant l'analyse du travail préalable) soit en moyenne 5 énoncés par transmission (c'est-à-dire par malade) et 3 énoncés par cible.

La répartition des énoncés en fonction des critères, Données ou Actions ou Résultats montrait que les informations concernant les données dominaient (44\%) comparativement aux actions (21\%) ou aux résultats $(15 \%)$. Restaient $20 \%$ d'énoncés inclassables dans ces trois catégories. Les données semblaient ainsi constituer les bases informationnelles de la relève.

\subsection{2.- La nature des cibles}

Sur 70 transmissions écrites $97 \%$ comportaient une (ou plusieurs) cible(s). Le nombre moyen de cibles par malade était de 1.6 (minimum 1 et maximum 5 cibles pour un malade). Ce résultat signifie que les infirmières qui ont participé à l'étude étaient significativement engagées dans la mise en œuvre du nouveau format de relève écrite.

Les cibles occurrentes communes aux trois services étaient peu nombreuses (" risque infectieux, comportement, douleur, élimination, examen, entré, sortant, devenir »). Celles communes à deux services s'étendaient un peu plus ("mobilisation, hyperthermie, atteinte cutanée, tension artérielle, alimentation, respiration, surveillance, hémodynamique »). Les cibles propres à un service étaient en relation avec la spécificité des pathologies du service médical concerné (en médecine: " confusion, dyspnée », par exemple; - en néphrologie: "éducation à la dialyse », par exemple; - en neurochirurgie: " pansement crâne », par exemple).

Compte tenu de cette disparité, nous avons réalisé une classification thématique des cibles. L'analyse a porté sur un échantillon de 1258 cibles. Cet échantillon correspondait au corpus des 70 transmissions écrites du jour de l'observation de l'activité des infirmières auquel s'ajoutait le corpus des transmissions écrites allant du jour de l'entrée du malade au jour de l'observation. Les critères du classement effectué ainsi que la répartition des cibles en fonction de chaque catégorie sont consignés dans le tableau 6.

\begin{tabular}{|l|c|}
\hline \multicolumn{1}{|c|}{ Critères de catégorisation des cibles } & Répartition en \% \\
\hline $\begin{array}{l}\text { Physiologie (concerne un événement physiologique qui survient: } \\
\text { écoulement nasal, dyspnée, vomissement, céphalées, etc.) }\end{array}$ & 57 \\
\hline Phase de l'hospitalisation (entrée, consultation, sortant, bilan, bloc, etc.) & 12 \\
\hline $\begin{array}{l}\text { Gestes techniques (à) réalisés (r) (mobilisation pour soins, pansement } \\
\text { crâne, etc.) }\end{array}$ & 10 \\
\hline Psychologie (confusion, refus de traitement, etc.) & 9 \\
\hline Pathologie du patient (insuffisance rénale, diabète, etc.) & 5 \\
\hline Matériel technique (de perfusion, chambre implantable, etc.) & 3 \\
\hline Traitement en cours (injection fraxiparine, éducation à la dialyse, etc.) & 3 \\
\hline Divers & 1 \\
\hline
\end{tabular}

Tableau 6 : Répartition (en \%) des types de cibles en fonction des catégories 
thématiques

On observe que ce sont d'abord les événements physiologiques nouveaux, puis les phases de l'hospitalisation, les gestes techniques et les aspects psychologiques enfin, qui constituaient le contenu des cibles. Quelles sont les fonctions de ces informations pour la relève? Quelles indications fournissent elles concernant la régulation du suivi du malade à réaliser à la suite de la relève? Afin de préciser cet aspect, nous avons réalisé deux types d'analyses. Dans la première, nous nous sommes centrés sur les formes syntaxiques et verbales utilisées par les professionnelles dans la rédaction des écrits. Dans la deuxième, nous avons systématiquement recherché les traces de l'activité de l'infirmière (diagnostics, régulations, préconisations). Ces analyses nous semblaient pouvoir constituer des indicateurs de l'activité réalisée, des diagnostics et des actions de régulation proposés.

\subsection{3.- Fonction des transmissions ciblées pour la relève.}

L'objectif de cette analyse est de préciser les fonctions des informations contenues dans les transmissions écrites.

En étudiant en premier lieu la syntaxe, nous avons pu distinguer trois catégories. La première, sujet - verbe - complément ( $s v c)$, correspondait à la syntaxe canonique de l'écriture de la phrase; la deuxième à une forme relativement abrégée, verbe - complément $(\mathrm{sv})$; la troisième enfin, constituait une restriction syntaxique limitée à l'ordre des mots (om). Cette dernière catégorie ne correspondait pas seulement à l'usage de formes elliptiques, mais plutôt à une syntaxe restreinte similaire au langage restreint décrit par Falzon pour l'oral $(1989,2004)$. La répartition des énoncés des transmissions écrites en fonction de ces catégories montrait que la structure $s v c$ représentait $22 \%$ des énoncés; la structure $s v 43 \%$; et om $35 \%$. Ces données indiquaient qu'un langage de type opératif bien connu à l'oral (Falzon, 1989, 2004) était ici utilisé à l'écrit, souvent restreint à l'ordre des mots : « sacrum rouge, latéralisation et frictions, selles à 20 heures »). Par ailleurs, à ces restrictions, économiques, sélectives et efficaces entre experts, s'ajoutait une très grande proportion de mots techniques.

En ce qui concerne les formes verbales en second lieu, nous avons réparti chaque énoncé selon le temps utilisé pour la formulation. Les résultats de cette répartition ont indiqué que l'utilisation du temps passé était majoritaire ( $47 \%$, passé composé, participe passé), suivi du présent ( $23 \%$ ) ou de phrases sans verbes (29\%). L'utilisation des temps futurs est beaucoup plus rare (6\% pour le futur simple, $6 \%$ pour l'impératif). Ce résultat suggère que les informations écrites étaient dirigées vers les actions passées ou présentes (59\%) et/ou les données (29\%), mais beaucoup moins souvent vers les actions à réaliser ( 6 à $12 \%$ ), les anticipations, c'est-à-dire les régulations à réaliser et les dysfonctionnements à réguler. Bien entendu, les informations passées sont aussi primordiales pour construire les décisions d'actions futures. Cependant, ces dernières décisions constituaient, comme nous l'avons montré lors de l'analyse de la relève orale, les indicateurs de l'activité de suivi de l'état du malade par les professionnelles.

En troisième lieu, quelles traces de l'activité de suivi pouvait-on trouver dans les transmissions écrites, tout d'abord dans le choix de la cible? Nous avons pu repérer deux types de cibles. Le premier s'apparentait à l'utilisation de catégories issues d'un modèle théorique classiquement enseigné dans la formation des infirmières: "la théorie des besoins ». Ce modèle utilise une catégorisation en termes des fonctions physiologiques et psychologiques générales vitales comme par exemple «respiration 》, "élimination 》, « communication ». Les écrits présentés figure 2 contiennent cette nomenclature: «mobilisation, hydratation, élimination, sommeil ». Ce type de cible plutôt formel ne fournit pas d'emblée (à lui seul) une information sur l'état ou l'évolution du malade. Le deuxième type de cible se rapportait plutôt à une variable d'évolution de l'état du patient: "fatigue », "inappétence », "hypoglycémie ». Cette distinction entre ces deux types de cibles peut s'appliquer à des cibles de nature physiologique mais aussi psychologique. Comme l'ont montré les données du tableau 7, les cibles de type besoin semblaient dominer dans le domaine physiologique, les variables d'état étaient massivement réservées au domaine psychologique. 


\begin{tabular}{|l|c|c|}
\hline & Cible de type Besoin & Cible de type variable d'état \\
\hline Domaine physiologique & 55 & 45 \\
\hline Domaine psychologique & 2 & 98 \\
\hline
\end{tabular}

Tableau 7 : Répartition (en \%) des types de cibles de type besoin ou de type état en fonction de leur domaine, physiologique ou psychologique

Par ailleurs, il est apparu que dans $80 \%$ des écrits analysés, cette formulation des cibles qui utilisait la nomenclature « des besoins » pouvait générer une ambiguïté sémantique concernant la cohérence entre l'information apportée par la cible et celle apportée par les données. Prenons quelques exemples. Dans l'écrit suivant: " mobilisation (cible). Après lever au fauteuil, mieux pour respirer mais très mal au dos (données) ", on peut penser que le terme " mobilisation » renvoyant à un besoin, est ambigu, ou peu adapté, pour désigner l'information importante concernant l'état du malade (correspondant selon la prescription DAR à la cible) qui réside dans des difficultés respiratoires et non dans l'action de mobilisation entreprise. Il en va de même dans les deux exemples suivants (tirés de la figure 2): dans l'écrit «Mobilisation (cible). Retrouvée debout à côté du lit après le départ de sa fille (Données) » l'information cruciale « retrouvée debout » n'est pas précisément désignée par la cible. Dans l'écrit « Hydratation (Cible). Retrouvée au pied du lit avec les jambes à travers la barrière. Dépiquée, perf. coulait dans le lit (Données) 》, l'information décrite par les données est aussi reflétée de façon ambiguë par la cible hydratation (qui correspond à un besoin général en eau). Ce type d'ambiguité pourrait être interprété comme relevant d'une difficulté des infirmières à trouver ou repérer une cible. Cela serait relativement étonnant de la part de professionnelles expertes. Une hypothèse alternative nous semble plausible: il pourrait s'avérer très difficile de nommer par un terme générique ou unique une cible reflétant des changements exprimés par des signes cliniques et/ ou des comportements. Dans ce cas, la formulation en termes génériques issue de la « théorie des besoins » correspondrait à un usage adaptatif, détourné de ce registre médical, correspondant ainsi à une catachrèse instrumentale langagière, (Rabardel, 1995) pour désigner des problèmes (ou leurs conséquences) survenus chez le patient: problèmes liés à la mobilisation, problèmes liés à l'hydratation etc. Bref, en utilisant ce type de formulation, les professionnelles cherchaient-elles à s'adapter à la prescription? Il se pourrait aussi que les professionnelles n'aient pu se construire une représentation claire et précise de la notion de cible prescrite dans le DAR. Ces deux dernières alternatives refléteraient une ambiguïté de la prescription elle-même. Enfin, les problèmes de cohérence entre cibles et données étaient bien plus fréquents lorsque la cible désignait un besoin (87\%) que lorsque la cible était formulée en termes de variable d'état $(52 \%)\left(\mathrm{Chi}^{2}=7,63, \mathrm{p}<.01\right)$. Ainsi, la variable état rend mieux compte de l'activité de l'infirmière. Mais surtout, les ambiguïtés référentielles que nous venons de relever témoignent de la difficulté à utiliser le format DAR. Les contraintes structurales de ce format ont conduit semble-t-il à des transformations des écrits peu compatibles avec les représentations fonctionnelles en termes de signes cliniques et psychologiques qui constituent les indicateurs de suivi des patients pour l'infirmière.

\begin{tabular}{|l|c|c|}
\hline \multicolumn{1}{|c|}{ Thèmes de référence } & Relève orale & Relève écrite \\
\hline Prescription médicale & 34 & 44 \\
\hline Comportement & 18 & 20 \\
\hline Signes cliniques & 16 & 20 \\
\hline Diagnostic & 9 & 6 \\
\hline Motif d'hospitalisation & 6 & 0 \\
\hline Identité & 4 & 0 \\
\hline Pathologies (de départ) & 4 & 0 \\
\hline Actions hors prescription & 4 & 8 \\
\hline Organisation générale & 4 & 2 \\
\hline
\end{tabular}

Tableau 8 : Répartition (en \%) des thèmes de la relève orale et de ceux de la relève écrite 
Des traces de l'activité se révélaient aussi dans la nature des informations développées. La distribution des différents types de thèmes développés dans les transmissions à partir des cibles, se rapprochait de celle relevée pour les transmissions orales, tableau 8.

Nous avons à nouveau observé une sensibilité importante à l'expertise de la répartition des thèmes. Comportements, signes cliniques et décisions d'actions (hors prescription médicale) apparaissaient plus élevés dans les deux groupes les plus expérimentés (respectivement: $63.5 \%$ pour les expertes, et $66 \%$ pour les expérimentées) que chez les novices (36.5\%). La tendance était inverse en ce qui concerne la prescription médicale qui apparaissait plus fortement chez les novices (50\%) que chez les infirmières expertes $(27.5 \%)$ ou expérimentées $(20 \%)$.

\subsection{4- La disparition des régulations en cours dans les transmissions écrites}

L'une des fonctions de la relève orale concernait la régulation des dysfonctionnements, en particulier les dysfonctionnements non régulés ou à réguler (51\% des dysfonctionnements). Dans les écrits, nous avons relevé exclusivement des régulations réalisées. Ainsi, les transmissions écrites ne contenaient aucune mention explicite des dysfonctionnements à réguler et des échecs de régulation. Paradoxalement, ces informations directement liées au suivi du malade, à l'anticipation de risques ont fait l'objet d'un filtrage entre la transmission orale et écrite. Certains types de régulations, qui pourraient être discutées lors de l'échange de la relève orale, ne pouvaient pas être aussi aisément explicités dans la relève écrite, du fait par exemple de l'incertitude de la situation à réguler et du possible manque de fiabilité des informations attachées à une régulation en cours. Un examen plus approfondi des données issues de l'analyse de l'activité préalable de soin que nous avons enregistrées pourrait fournir des informations pertinentes pour trouver les raisons de cette disparition des régulations.

En résumé, nous avons observé que les transmissions écrites actuelles en DAR n'étaient pas adaptées aux besoins des infirmières. Elles contenaient, néanmoins, une large part des traces de l'activité spécifique de suivi du malade par l'infirmière: évolution des signes cliniques, des comportements, actions autonomes hors prescriptions. Mais un filtrage important était opéré en ce qui concerne les régulations, enjeu majeur des échanges de la relève orale. De plus, les informations décrites étaient relativement différentes en fonction de l'expertise. Finalement, les deux types de relève, écrite et orale ne semblaient pas remplir la même fonction; la relève écrite ne véhiculait pas les mêmes informations que la relève orale et ne concernait pas forcément le même destinataire que la relève orale.

\subsection{5.- Les critères de tri dans la relève écrite}

Quels sont les critères cognitifs de la sélection opérée à l'écrit? C'est ce que nous allons aborder maintenant en exposant, en dernière analyse, les résultats obtenus à partir des protocoles verbaux de chaque infirmière concernant chacune de ses transmissions écrites au cycle précédent.

Lors d'une tâche d'explicitation individuelle (Bisseret, Sébillotte, \& Falzon, 1999), chaque infirmière avait sous les yeux d'une part, les transmissions tout dernièrement écrites et d'autre part, la retranscription de la base de connaissances référentielles précédemment analysée pour chaque malade. Chaque infirmière devait répondre à deux questions (standardisées) pour chaque transmission: "Comment avez-vous fait pour sélectionner (choisir) les informations présentes dans la transmission? » (ainsi de suite pour chaque transmission) et " comment avez-vous fait pour décider de ne pas transmettre (choisir) certaines des informations? ». L'objectif de cette épreuve était de recueillir les règles « explicites » utilisées au cours de la phase de rédaction elle-même. Pour chaque transmission, nous avons tout d'abord considéré les mécanismes de tri explicitement évoqués communs aux trois groupes d'infirmières et à chaque niveau d'expertise, puis nous avons distingué plus précisément les facteurs de tri positifs correspondants à l'information retenue, et enfin les facteurs de tri négatifs correspondants à l'information écartée. Dans chaque cas, une seule évocation explicite du critère était suffisante pour qu'il soit retenu. 
En premier lieu, la totalité des professionnels a indiqué l'existence d'une étape de recherche en mémoire, accompagnée ensuite d'autres activités. Plus précisément, les expertes déclaraient se baser sur les indicateurs suivants: "chercher dans sa mémoire les informations du suivi", "lecture des transmissions précédentes 》, "interactions avec la malade »; les novices ont fait état de: "chercher dans sa mémoire les informations ", "les informations se présentent sous la forme d'images »; les expérimentées indiquaient: " chercher dans sa mémoire les informations sur le suivi », "lecture des transmissions précédentes ». Au cours de cette phase, commune à tous, on peut ajouter que les novices recherchaient plutôt des informations fondées sur les soins réalisés ( «images »), les expertes fondant plutôt leur évaluation sur les informations issues de la supervision de l'état du malade.

En second lieu, nous avons relevé les critères de sélection des informations plus spécifiques que les infirmières déclaraient utiliser au cours de la phase de rédaction elle-même de la transmission, et cela en fonction de chaque niveau d'expertise. Les résultats sont consignés dans les tableaux 9a (informations retenues) et $9 \mathrm{~b}$ (informations écartées).

\begin{tabular}{|c|c|c|}
\hline Novices & Expérimentées & Expertes \\
\hline $\begin{array}{l}\text { Buts du tri déclarés } \\
\text { Prescription du médecin (à réaliser). } \\
\text { Ne pas répéter les informations } \\
\text { Intégrer le « diagnostic infirmier. }\end{array}$ & $\begin{array}{l}\text { Buts du tri déclarés } \\
\text { Repérer catégoriser les difficultés. }\end{array}$ & $\begin{array}{l}\text { Buts du tri déclarés } \\
\text { Choisir des connaissances } \\
\text { mémorisées sur le malade } \\
\text { concernant l'évolution de son } \\
\text { état. }\end{array}$ \\
\hline $\begin{array}{l}\text { Critères de sélection } \\
\text { Problème du malade (diagnostic } \\
\text { initial). } \\
\text { Apparition d'un problème le jour } \\
\text { même. } \\
\text { Changement pendant le poste. } \\
\text { Echange avec le médecin en rapport } \\
\text { avec la prescription. } \\
\text { Tâche du poste non réalisée en } \\
\text { rapport avec la prescription. } \\
\text { Etat mesuré (fiches) de la } \\
\text { pathologie. }\end{array}$ & $\begin{array}{l}\text { Critères de sélection } \\
\text { Informations non ciblées sous forme } \\
\text { de bilan (fiches etc.). } \\
\text { Evolution d'un paramètre (y } \\
\text { compris hors diagnostic initial). } \\
\text { Compléments d'informations } \\
\text { par rapport à la cible antérieure } \\
\text { (mesures, signes cliniques) sous } \\
\text { forme de résultats. }\end{array}$ & $\begin{array}{l}\text { Critères de sélection } \\
\text { Evolution des données } \\
\text { cliniques par rapport au } \\
\text { dernier poste occupé. } \\
\text { Changements importants dans } \\
\text { la prescription qui entraînent } \\
\text { un risque vital (suivi des } \\
\text { infirmières du poste à venir). } \\
\text { Informations signalant } \\
\text { un doute sur les effets du } \\
\text { traitement. } \\
\text { Résultats des examens réalisés } \\
\text { Actions autonomes réalisées. }\end{array}$ \\
\hline
\end{tabular}

Tableau 9a : Critères de sélection des informations à écrire en fonction du niveau d'expertise.

En ce qui concerne les informations retenues (tableau 9a) il est apparu que les professionnelles novices sélectionnaient plutôt des informations ciblées sur la tâche prescrite: prescription médicale, répétitions, tâches du poste non réalisées. Les professionnelles un peu plus expérimentées s'appuyaient surtout sur la sélection d'informations absentes de la transmission précédente ou des autres fiches écrites existantes. C'est ce que faisaient également les expertes dans une certaine mesure. Mais ces dernières semblaient surtout sélectionner les informations pertinentes à partir des représentations mémorisées lors des postes précédents et surtout de l'activité déployée au cours du cycle de travail. Ces informations concernaient un modèle de l'évolution de l'état du malade construit à travers l'activité de supervision, sur la base des signes cliniques et comportementaux qui étaient explicites, précis et concernaient la dimension du risque pour le patient: évolution de données cliniques, changements dans la prescription, information signalant un doute sur les effets du traitement. 


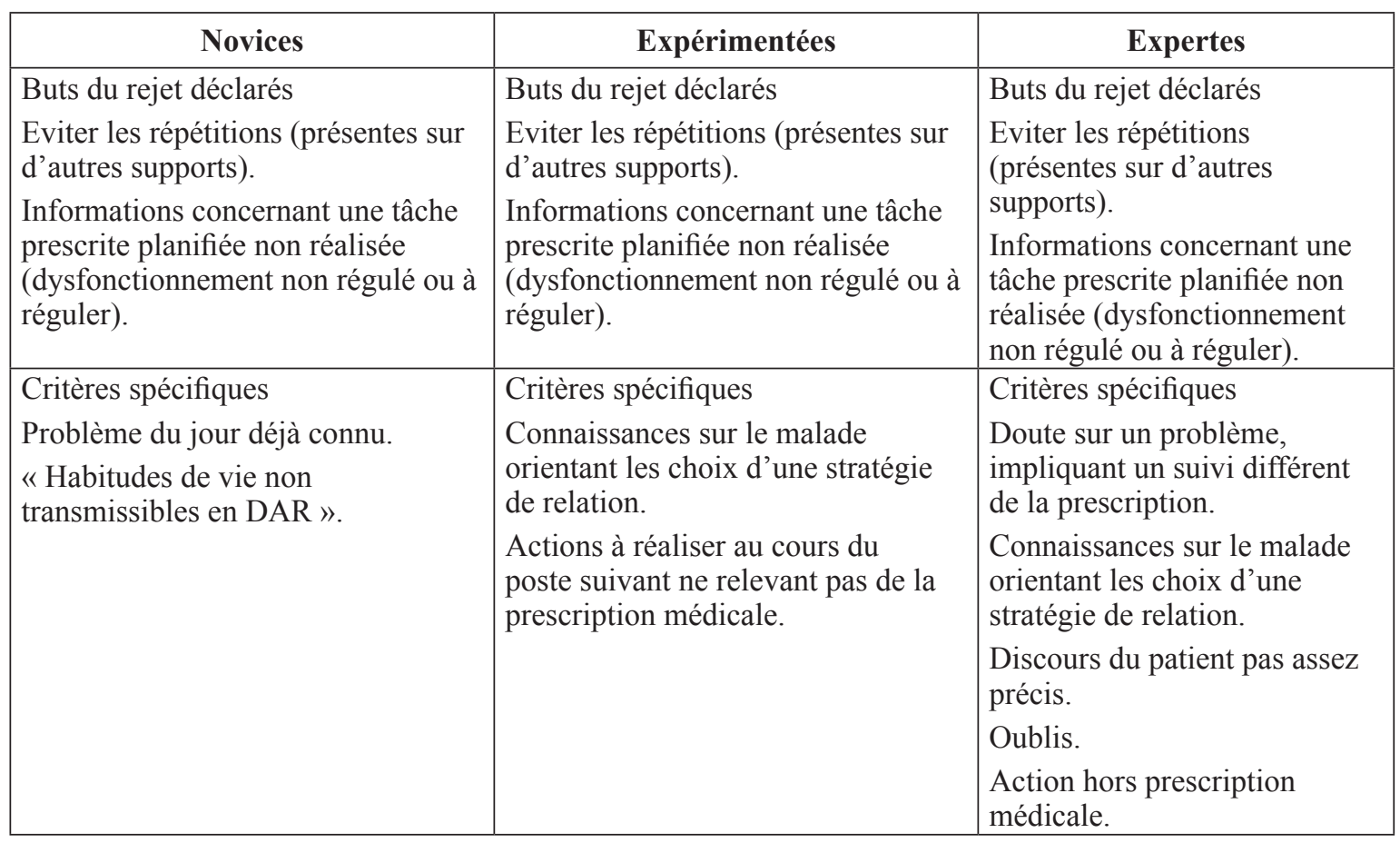

Tableau 9b: Critères de rejet des informations lors de la phase de rédaction

En ce qui concerne les critères de rejet des informations (tableau $9 \mathrm{~b}$ ), notons que la redondance des informations est apparue pour les trois sous-groupes mais également et surtout les actions ou dysfonctionnement à réguler ou non régulés. Chez les expertes, il semblait que lorsqu'un doute apparaissait (à propos de la signification des signes cliniques) l'information n'était pas transcrite. Ainsi les transmissions écrites n'explicitaient pas les situations à réguler, non régulées ou pour lesquelles un doute trop important subsistait. Ces situations constituaient plutôt des activités de résolution de problème qui sont au centre des échanges de la relève orale.

\section{4.- Discussion et conclusion}

Au cours d'une démarche comparative entre trois phases de l'activité d'infirmières comprenant l'activité de soins puis successivement les relèves orales et écrites, nous avons réalisé une évaluation ergonomique de l'usage d'un format de transmission écrite nouvellement prescrit: les transmissions ciblées en DAR. Cette étude a concerné 9 infirmières, expertes, expérimentées, et novices, travaillant avec 70 patients résidants dans trois services de médecine comparables. L'analyse a montré que, d'une part, la forme de ces transmissions écrites ne correspondait que très minoritairement à la structure prescrite DAR, et d'autre part que les activités de régulation en cours ou posant problème (doutes à propos d'un signe, échecs de régulations) particulièrement importants pour la relève avaient été écartées de l'écrit alors qu'elles étaient le plus souvent l'enjeu de la relève orale. Ainsi, le dialogue permis à l'oral et les traces laissées par l'écrit possèdent des rôles différents dans le suivi du patient au cours des différents postes. Dans la relève, oral et écrit contenaient des traces du travail de supervision de l'état des malades par les infirmières. Cette activité de supervision reposait sur la sélection, la transmission et la discussion d'indicateurs spécifiques : modification ou évolution des signes cliniques et des comportements. Ces indicateurs, sensibles à l'expertise, permettaient la prévention et la régulation des dysfonctionnements et donc des risques éventuels.

À partir du bilan des données recueillies au cours des trois phases d'analyse que nous avons suivies, nous avons élaboré une synthèse sous la forme d'une modélisation de l'activité de transmission écrite étudiée ici. Cette modélisation est inspirée d'une part, des modèles de la production écrite élaborés en psychologie cognitive qui sont apparus très compatibles avec nos résultats (Fayol, 1997) et d'autre 
part, des résultats de notre analyse des productions écrites des relèves. Le modèle proposé figure 4 est composé de trois étapes. Au cours de la première, l'infirmière à pour but de choisir une cible. Pour cela, elle recherche des informations pertinentes et saillantes en mémoire concernant l'évolution du patient et les activités réalisées au cours de la période précédente. Cette recherche pourrait être effectuée en parallèle avec un autre traitement qui correspondrait plutôt à un tri des informations à plusieurs critères. En effet, il faut choisir une donnée ou une action nouvelle importante pour la régulation du suivi du patient, comme cible, en la comparant non seulement aux écrits antérieurs, mais aussi aux « normes internes d'acceptabilité » notamment en cas de dysfonctionnement non régulé (en sachant que les informations sur ces régulations peuvent être transmises à l'oral). Cette tâche implique une activité de décision. Cette première étape conduit à l'élaboration d'une représentation conceptuelle multidimensionnelle des éléments choisis, correspondant à une cible potentielle.

La deuxième étape est une phase de planification et d'organisation des informations en Données et/ ou Action et/ou Résultats, contraignant à tenter de mettre au point une structure textuelle compatible avec le formalisme.

La dernière étape correspond à la mise en mots (vocabulaire, formulations, y compris implicites ou codées). Comme l'ont montré (dans d'autres domaines de l'écrit) les résultats des travaux en psychologie expérimentale sur la production écrite (Bock \& Cutting, 1992; Bonnin \& Fayol, 1996; Fayol, 1997; Alamargot, \& Dansac, 2003) le « goulot d'étranglement» de la transmission que constitue la transformation des représentations issues de l'activité en DAR (deuxième étape) pourrait s'avérer difficile et coûteux pour l'opérateur. L'infirmière pourrait être amenée à chercher des précisions complémentaires, nécessitant un nouveau contact avec la représentation issue de l'activité, c'est-àdire les connaissances préalables concernant le patient et les actions réalisées. De telles rétroactions (cycles) sont représentées figure 4 par les doubles flèches verticales du modèle. L'éventuel coût peut poser le problème de l'utilisation réelle, à long terme au cours de l'activité, du formalisme prescrit. Sur la durée, un risque d'abandon spontané ou à tout le moins de transformation de ce système de notation des informations sont à anticiper. Quel est le gain pour l'opérateur de l'utilisation de ce formalisme, en terme de production écrite et d'usage?

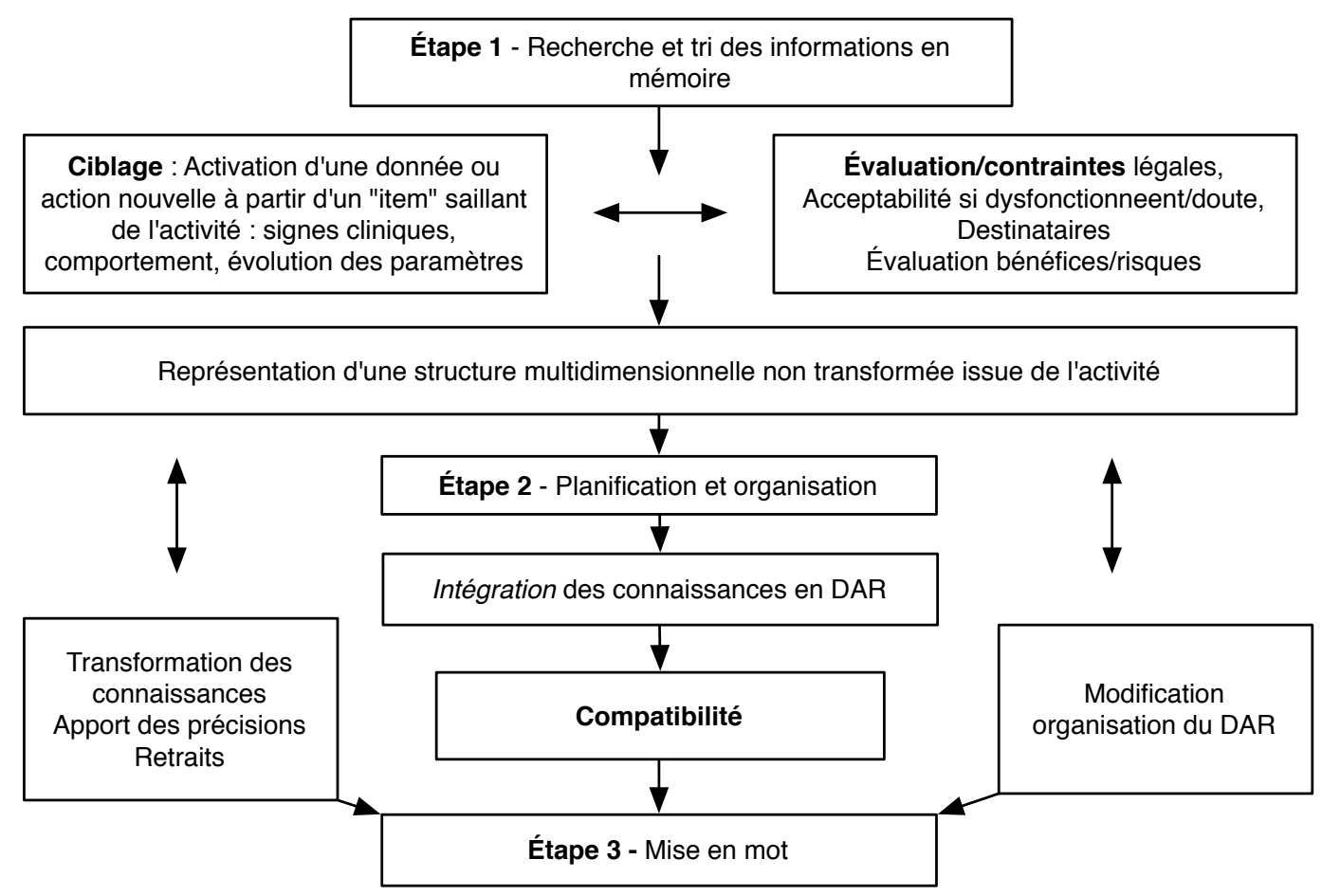

Figure 4 : Un modèle de la production écrite des transmissions en Données, Actions et Résultats 
Il ne faudrait pas que les contraintes d'écriture deviennent, pour l'opérateur, la tâche prioritaire par rapport à la tâche de transmission des informations pertinentes concernant le suivi de l'évolution de l'état du malade, qui deviendrait secondaire. Par exemple, qu'une information importante (un indice précurseur) soit rejetée parce que n'entrant pas dans le formalisme.

Nous avions pour objectif d'évaluer l'ergonomie du nouveau format d'écriture, la transmission ciblée formalisée en DAR, pour son usage dans la relève écrite chez les infirmières en poste. Ce nouvel outil avait été proposé, puis mis en œuvre, par l'institution hospitalière dans le cadre de la conduite de l'accréditation de l'établissement. Les bénéfices attendus « a priori » de cette nouvelle prescription par les commanditaires étaient de permettre un meilleur suivi de l'état du malade en sélectionnant les aspects significatifs de l'évolution du patient, d'obtenir une traçabilité des transformations du patient, de capitaliser des informations écrites fiables et pratiques pour assurer des soins continus. Cependant aucune analyse préalable de l'activité de transmission orale et écrite, des compétences existantes chez les professionnelles dans ce domaine, n'avait été projetée ni réalisée par l'institution avant l'introduction de ce nouvel outil. Une telle analyse aurait pu permettre de mieux comprendre le fonctionnement de relèves avant d'introduire un outil «d'amélioration » des écrits. Devant les difficultés objectives liées à la mise en œuvre des transmissions ciblées, c'est une formation professionnelle qui avait été envisagée et qui avait fait l'objet de la demande qui nous avait été adressée. Or, notre analyse a révélé que cette réponse pédagogique n'est pas la première indiquée. En effet, nous avons montré que l'outil préconisé n'était pas adapté aux besoins des professionnelles. En effet, loin de constituer un " outil cognitif » pour l'activité de rédaction des transmissions écrites (Rogalski, 2001), il est apparu que, en l'état, le format Cible + DAR était contraignant, très faiblement utilisé, peu adapté aux besoins des infirmières et difficile à mettre en œuvre. Les processus de filtrage des informations entre l'activité réalisée avant la relève et la relève écrite, que nous avons ici analysés, montrent que les relèves orale et écrite ne contiennent pas les mêmes traces et n'ont pas le même rôle dans le suivi du patient. L'enjeu de la relève orale ne semble pas seulement de transmettre les informations nouvelles concernant le patient mais d'échanger à propos de dysfonctionnements en cours de régulation ou non régulés et donc de prévenir les risques pour le patient. Les échanges à propos de ces dysfonctionnements pourraient constituer de véritables outils d'un suivi continu des patients. Or, avec l'introduction des nouvelles normes d'écriture, leur évocation disparait totalement des traces écrites. Si ces transmissions écrites ciblées conservent des traces de l'activité de supervision de l'infirmière à travers la description des signes cliniques et des comportements nouveaux des patients, elles se limitent à la description des dysfonctionnements régulés. On peut même se demander jusqu'à quel point l'introduction du nouveau format d'écriture n'a pas dégradé les pratiques de transmission écrites existantes?

Si une optimisation de l'organisation et de la précision de transmissions écrites semble nécessaire, et surtout souhaitée par les professionnelles de l'institution, il apparait que la structure d'un tel écrit devrait se rapprocher de la structure de l'activité de supervision de l'infirmière et de l'organisation des représentations opératives issues de cette activité. Par ailleurs, le choix de la structure de l'écrit, comme celui des critères de contenu (saillance) et de précision des informations pourrait faire l'objet d'un travail de co-conception par les professionnelles et les prescripteurs de l'institution hospitalière, dans le cadre du développement des compétences dans le domaine de la relève. Il apparaît notamment nécessaire de connaître précisément les besoins réels des infirmières pour optimiser les écrits. Cela semblerait particulièrement utile pour les infirmières novices comme l'a montré notre comparaison systématique entre les trois niveaux d'expertise.

Dans cette perspective, une analyse approfondie des transmissions écrites « spontanées » des professionnelles serait à réaliser dans le but de mieux comprendre leur fonctionnement, et cela en dehors de toute modification de format. Les résultats présentés précédemment indiquent également qu'il apparait nécessaire de conduire un travail de définition de la nature et de la forme des écrits attendus. Ce travail pourrait utilement s'orienter vers des formats plus proches des représentations « dynamiques opératives » de l'état du malade construites au cours de l'activité par les infirmières. En effet ces 
représentations apparaissent «multidimensionnelles » par exemple, en même temps que des signes cliniques « objectifs » ou mesurables elles intègrent le « vécu » du patient. Enfin, une réflexion sur le contenu des informations relativement à leur fiabilité pourrait être utilement engagée. En effet, les relèves, la transmission (écrite ou orale) ne constituent-elles pas une part très critique de la tâche prescrite à l'opérateur montant par l'opérateur descendant, et en cela une activité cruciale pour la continuité des soins?

\section{RÉFÉRENCEMENT}

Boucheix, J.-M \& Coiron, M. (2008). Analyse de l'activité de transmission écrite au cours des relèves de poste à l'hôpital: Évaluation ergonomique de l'usage d'un nouveau format d'écriture. @ctivités, 5 (1) pp. 79-102, http://www.activites.org/v5n1/v5n1.pdf

\section{RÉFÉRENCES}

Alamargot, D., \& Dansac, C. (2003). Composer une consigne à partir de sources: ce que nous indiquent les activités graphiques et oculaires des rédacteurs. Journées d'études, Production, compréhension et usages des écrits techniques au travail. MSHS, Toulouse, 19-20 juin.

Bisseret, A., Sébillotte, S., \& Falzon, P. (1999). Manuel pratique pour l'analyse des activités expertes. Toulouse: Octarès.

Bock, J.K., \& Cutting, J.C. (1992). Regulating mental energy: performance units in language production. Journal of memory and language, 31, 99-127.

Bonnin, P., \& Fayol, M. (1996). L'étude en temps réel de la production du langage écrit: Pourquoi et comment. Etudes de Linguistique Appliquée, nº101, 8-19.

Boucheix, J.M. (2003). Ergonomie et formation: approche d'ergonomie cognitive de l'apprentissage professionnel. Psychologie Française, 48-2, 43-56.

Boucheix, J.M. (2005). L'accueil, orientation courtoise ou activité de résolution de problèmes, In M. Cerf, \& P. Falzon, Relations de service: Travailler avec le client (pp.87-111). Paris: PUF.

Bourdin, B. (1994). Coût cognitif de la production verbale. Etude comparative orallécrit chez l'enfant et l'adulte. Thèse pour le doctorat en psychologie de l'Université de Bourgogne.

Boutet, C. (1995). Les écrits professionnels. Sociologie du Travail, 4, 25-52.

Cellier, J.M., De Keyser, V., \& Valot, C. (1996). Le temps dans la conduite des systèmes dynamiques. Paris: PUF.

Cosnier, J., Grosjean, M., \& Lacoste, M. (1993). Soins et communications, Lyon: PUL.

Cerf, M., \& Falzon, P. (2005). Ergonomie de la relation de service: travailler avec le client. Paris PUF.

Cerf, M., Valléry, G, \& Boucheix, J.M. (2004). Les situations de service: enjeux et développement. In P. Falzon (Ed.), Traité d'Ergonomie (chapitre 34, pp. 565-581). Paris: PUF.

Davillerd, C., \& Grusenmeyer, C. (1994). La relève de poste: une période spécifique de travail collectif. Actes du XXVIIéme Congrès de la société d'ergonomie de langue Française (pp. 143-151). Toulouse: Octarès.

Errard, G., Kostulski, K., \& Trognon, A. (1996). Le dossier de soins : instrument de communication. A. A. R. C. $H$.

Falzon, P. (1989). Ergonomie cognitive du dialogue. Grenoble: PUG.

Falzon, P. (2004). Traité d‘Ergonomie. Paris: PUF

Fayol, M. (1997). « Des idées au texte: psychologie cognitive de la production écrite » Paris: P.U.F.

Giboin, A. (2004). La construction des référentiels communs dans le travail coopératif. In J.M. Hoc, \& F.

Darses (Eds.). Psychologie ergonomique: tendances actuelles (pp. 119-139). Paris: PUF. 
Grosjean, M., \& Lacoste, M. (1999). Communication et Intelligence Collective: Le Travail à l'Hôpital. Paris: PUF.

Grusenmeyer C. (1995). Interaction langagière et représentation mentale partagée. Une étude de la relève de poste. Psychologie Française, 40, 47-59.

Grusenmeyer C. (1996). De l'analyse des communications à celle des représentations fonctionnelles partagées. Thèse de Doctorat de Psychologie Cognitive, INRS, A.8/1. 008.

Grusenmeyer, C., \& Trognon, A. (1996). Strutures of natural reasoning within functional dialogues. Pragmatics \& Cognition, 4 (2), 305-346.

Hayes, J.R ., \& Nash, J.G. (1996). On the nature of planning in Writing. In C.M. Levy, \& S. Randsell (Eds.), The science of writing (pp. 29-55). Mahwah, N.J.: Lawrence Erlbaum Associates.

Heurley, L. (1994). Traitement des textes procéduraux. Etude de psycholinguistique cognitive des processus de production et de compréhension chez des adultes non experts. Thèse pour le doctorat de Psychologie, Université de Bourgogne.

Hoc, J.M. (1996). Supervision et contrôle de processus. La cognition en situation dynamique. Grenoble: PUG.

Montmollin de, M., (1986). L'intelligence de la tâche. Bern: Peter Lang.

Leplat, J. (1997). Regards sur l'activité en situation de travail. Paris: PUF, Le Travail Humain.

Levelt, W.J.M. (1989). Speaking: From intention to articulation, Cambridge: MA, MIT Press.

Levelt, W.J.M., \& Maassen, B. (1991). Lexical search and order of mention in sentence production. In W. Klein, \& W.J. levelt (Eds.), Crossing boundaries of linguistics (pp. 109-123). Dordrecht: Reichel.

Pastré, P. (1997). Didactique professionnelle et développement. Psychologie Française, 42, 89-10.

Rabardel, P. (1995). Les hommes et les Technologies. Approche Cognitive des Instruments Contemporains. Paris: Armand Colin.

Raufaste, E. (2001). Les mécanismes cognitifs du diagnostic médical. Optimisation et expertise. Paris: PUF.

Rogalski, J. (2001). Connaissances opérationnelles, compétences. In J. Leplat, \& M. de Montmollin (Eds.), Les compétences en ergonomie (pp. 250-275). Toulouse: Octarès Editions.

Rogalski, J. \& Samurçay, R. (1994). Modélisation d'un savoir de référence et transposition didactique dans la formation des professionnels de haut niveau. In G. Arsac, Y. Chevallard, J.L. Martinand, \& A. Tiberghien (Eds.). La transposition didactique à l'épreuve (pp. 35-71). Grenoble: La Pensée Sauvage Editions.

Vallery, G., Boucheix, J.M., Leduc, S. \& Cerf, M. (2004). Analyser les situations de service: questions de méthodes. In M. Cerf, \& P Falzon (Eds.). Situations de service: travailler dans l'interaction, (pp. 21-40). Paris: PUF.

RÉSUMÉ

L'objectif du travail présenté dans cet article est d'évaluer l'adaptation ergonomique d'un nouveau format de rédaction des transmissions écrites, prescrit par l'hôpital (CHU), aux besoins des infirmières lors de la relève de poste. L'étude se centre en particulier sur la nature du filtrage de l'information opérée dans les transmissions. Une démarche en trois temps a été suivie auprès d'une population de 9 infirmières en charge de 70 patients. : (1) analyse l'activité des soins auprès des patients, préalable à la relève, (2) analyse la relève orale des infirmières descendantes et montantes, (3) analyse de la relève écrite consécutive des infirmières descendantes. Les résultats montrent que le nouveau format d'écriture n'est pas adapté aux besoins des professionnelles. En particulier, les indicateurs cruciaux, signes cliniques et comportements, utilisés par les infirmières au cours de la relève orale pour la supervision de l'évolution de l'état des patients tendent à disparaître de la transmission écrite. Les régulations des dysfonctionnements de l'état du patient qui sont un enjeu majeur des échanges de la relève orale sont absentes des transmissions écrites. Le dialogue de l'oral et les traces laissées 
par l'écrit possèdent des rôles différents dans le suivi du patient. On discute enfin d'un modèle proposé de production écrite puis de préconisations en vue de la conception « d'outils » pour l'optimisation des activités de relève à l'hôpital.

\section{Mots CLÉS}

Relève de poste, transmissions orales et écrite, format d'écriture, modèle de production.

\section{RESUMEN}

El objetivo del trabajo presentado en este artículo es el de evaluar la adaptación ergonómica de un nuevo formato de redacción de las transmisiones escritas, prescripto por el hospital universitario, a las necesidades de las enfermeras durante los relevos de puesto. El estudio se centra especialmente en la naturaleza del filtro de la información que se opera durante las transmisiones. Se ha seguido una metodología organizada en tres tiempos a través de la cual se analizó a una población de 9 enfermeras a cargo de 70 pacientes: (1) análisis de la actividad relacionada con el cuidado de los pacientes, previa al relevo, (2) análisis del relevo oral de las enfermeras que « se bajan » y de las que « se suben », (3) análisis del relevo escrito consecutivo de las enfermeras que « se bajan ». Los resultados muestran que el nuevo formato de escritura no se adapta a las necesidades de las profesionales. En particular, los indicadores cruciales, signos clínicos y comportamientos, utilizados por las enfermeras a lo largo del relevo oral para supervisar la evolución del estado de los pacientes tienden a desaparecer de la transmisión escrita. Las regulaciones de las disfunciones del estado del paciente, que consisten en un desafío muy importante de los intercambios durante el relevo oral, también están ausentes de las transmisiones escritas. Los roles del diálogo oral y de las huellas de lo escrito son diferentes en cuanto al seguimiento del paciente. Finalmente, se discutirá un modelo de producción escrita propuesto, para terminar con las recomendaciones en vistas del diseño de « herramientas » para la optimización de las actividades de relevo en el hospital.

Palabras Clave

Relevo de Puesto, transmisiones orales y escritas, formato de escritura, modelo de producción

Article soumis le 14 mai 2007 accepté pour publication le 23 octobre 2007 